\title{
Estructura Interna del Cognitive Emotion Regulation Questionnaire (CERQ-18) en una Muestra de Adolescentes Peruanos
}

\author{
Internal Structure of the Cognitive Emotion Regulation Questionnaire (CERQ-18) in \\ a Sample of Peruvian Adolescents
}

\author{
Jhonatan S. Navarro-Loli ${ }^{1}$, Sergio Dominguez-Lara ${ }^{2}$ y Leonardo A. Medrano ${ }^{3}$
}

\begin{abstract}
Resumen
El presente estudio tuvo como objetivo identificar las evidencias de validez de la estructura interna y en relación con otras variables del Cognitive Emotion Regulation Questionnaire (CERQ-18) en adolescentes peruanos. La muestra fue de 255 adolescentes entre los 13 y 19 años $\left(M_{\text {edad }} 15.494, D E_{\text {edad }}=1.192\right)$ de una Institución Educativa pública del distrito de Ventanilla (Perú). Los instrumentos utilizados fueron el CERQ18 y el Inventario de Depresión Estado/Rasgo. Se plantearon cuatro hipótesis estructurales que fueron evaluados mediante un análisis factorial confirmatorio, incluyendo análisis bifactor. Los resultados indican que el modelo evolucionista representadas por las estrategias automáticas (autoculpa, rumiación, catastrofización) y elaborativas (aceptación, focalización en los planes, reinterpretación positiva, focalización positiva y poner en perspectiva), presentan indicadores satisfactorios, favoreciendo su interpretación, y mostrando relaciones significativas con afecto positivo y negativo. Se concluye que la estructura interna del CERQ-18 en adolescentes es respaldada por el modelo evolucionista.
\end{abstract}

Palabras clave: estrategias cognitivas de regulación emocional, CERQ, validación, adolescentes

\begin{abstract}
The present study aimed to identify the evidence of validity of the internal structure and in relation to other variables of the Cognitive Emotion Regulation Questionnaire (CERQ-18) in peruvian adolescents. The sample consisted of 255 adolescents between the ages of 13 and $19(\mathrm{M}=15.494, \mathrm{DE}=1.192)$ from a public school of the district of Ventanilla (Peru). The instruments used were the CERQ-18 and the State / Trait Depression Inventory. Four structural hypotheses were proposed, which were evaluated by confirmatory factor analysis, including bifactor analysis. The results indicate that the evolutionary model represented by automatic (self-blame, rumination, catastrophization) and deliberate strategies (acceptance, refocus on planning, positive reappraisal, positive refocusing and putting into perspective), present satisfactory indicators, favoring their interpretation, and showing significant relationships with positive and negative affect. It is concluded that the internal structure of CERQ-18 in adolescents is supported by the evolutionary model.
\end{abstract}

Keywords: cognitive emotion regulation strategies, CERQ, validation, adolescents

\footnotetext{
${ }^{1}$ Maestro en Psicología Clínica. Docente y Investigador. Instituto de Investigación de la Escuela de Psicología de la Universidad de San Martín de Porres, Lima, Perú. Av. Tomas Marsano 242 (5to piso), Surquillo, Perú. Tel.: 51-1-5136300, anexo 2186.

Correo: jnavarrol1@usmp.pe; jhonatan_navarro1602@yahoo.es

${ }^{2}$ Maestro en Psicología Clínica y de la Salud. Docente y Investigador. Instituto de Investigación de la Escuela de Psicología de la Universidad de San Martín de Porres, Lima, Perú. Av. Tomas Marsano 242 (5to piso), Surquillo, Perú. Tel.: 51-1-5136300, anexo 2186. Correo: sdominguezmpcs@gmail.com

${ }^{3}$ Doctor en Psicología. Secretario de Investigación, Docente y Investigador. Universidad Siglo 21, Córdoba, Argentina. Facultad de Psicología, Universidad Nacional de Córdoba, Argentina. Del Cid 321 (CP: 5000). Villa Allende, Argentina. Tel.: 0054-0351-

153874871. Correo: leomedpsico@gmail.com

Revista Iberoamericana de Diagnóstico y Evaluación - e Avaliação Psicológica. RIDEP · No54 · Vol.1 · 165-178 · 2020

ISSN: 1135-3848 print /2183-6051online
} 


\section{Introducción}

La regulación emocional (RE) involucra cualquier proceso explícito o implícito que pueda alterar la emoción sentida, su duración y/o expresión (Denny, Silvers, \& Ochsner, 2009). Dentro de los diferentes factores involucrados en la RE, los procesos cognitivos poseen un rol destacado (Garnefski \& Kraaij, 2007). Para Garnefski, Kraaij y Spinhoven (2001) pueden reconocerse al menos nueve estrategias de regulación cognitiva (ERC): culpar a otros, referido a pensamientos que atribuyen la culpa del evento a otros; rumiación, que consiste en pensar constantemente en los sentimientos o problemas asociados el evento negativo; catastrofización, asociado a pensamientos que enfatizan la gravedad del evento; autoculpa, que son pensamientos donde la persona se atribuye la responsabilidad del evento; aceptación, conformado por pensamientos de aceptar lo acontecido y vivenciar las emociones $\sin$ juzgarlas; focalización en los planes, que son pensamientos relacionados con los pasos necesarios para la solución del problema; reinterpretación positiva, que consiste en asociar pensamientos positivos al evento displacentero; focalización positiva, que se basa en mantener pensamientos positivos y poner en perspectiva, que es comparar la gravedad del evento con otros para disminuir su impacto.

A pesar de que estas estrategias son usadas por las personas en diferentes etapas de la vida, particular atención merece las ERC en la población adolescente por dos motivos: a) este grupo se ve expuesto a diversos cambios y eventos relevantes, que, aunque son esperables, la magnitud de los mismos conlleva cierto riesgo psíquico (Buernett \& Blakmore, 2009; SeiffgeKrenke, 2000); b) durante el desarrollo de la adolescencia las estructuras corticales encargadas de funciones ejecutivas continúan en desarrollo, por lo que aún no disponen de los recursos necesarios para inhibir impulsos o desarrollar procesos cognitivos elaborativos más complejos (Giedd, 2008; Kuhn, 2006) que se evidencia en bajos niveles de autorregulación (Raimundi, Molina, Hernández-Mendo, \& Schmidt, 2017). La conjunción de ambos factores lleva a que se vea aumentada la vulnerabilidad psicológica durante este período evolutivo. En efecto, el uso de las ERC disfuncionales puede llevar a que el impacto de muchos cambios esperables durante esta etapa genere malestar psicológico y aumente la probabilidad de desarrollar trastornos mentales, lo que se refleja en la prevalencia de ansiedad y depresión en adolescentes, que es de $3.2 \%$ y $6.2 \%$ respectivamente (Erskine et al., 2016).

Diversos estudios sugieren que el impacto de eventos estresantes se encuentra mediado en gran medida por las ERC (Kraaij et al., 2003; Medrano, Muñoz-Navarro, \& Cano-Vindel, 2016), lo cual explicaría el hecho de que dificultades relacionadas con las ERC se asocien con el desarrollo de problemas internalizantes y externalizantes (Garnefski, Kraaij, \& Etten, 2005). En este sentido, existen ERC como la aceptación y la focalización positiva que están vinculadas a aspectos saludables del comportamiento humano como, por ejemplo, la resiliencia (Wante, Van Beveren, Theuwis, \& Braet, 2017) y la salud mental general (Raes, Griffith, Van der Gucht, \& Williams, 2014). Por el contrario, otras ERC se vinculan a conductas desadaptativas. Tal es el caso de la rumiación, catastrofización y autoinculpación, que se asocian con problemas en el funcionamiento social (Mihalca \& Tarnavska, 2013), depresión (Abela \& Hankin, 2011; D'Acremont \& Van der Linden, 2007; Garnefski et al., 2005; Garnefski \& Kraaij, 2006, 2016; Nolen-Hoeksema, Wisco, \& Lyubomirsky, 2008), ansiedad (Legerstee, Garnefski, Jellesma, Verhulst, \& Utens, 2009), violencia física y verbal (Pen, Jin, We-Han, Xiu-Hong, \& Yu-Sui, 2016), obesidad (Tan, Xin, Wang, \& Yao, 2018), conductas sexuales de riesgo (Yu-Ning, ShuQuiao, \& Xiu-Hong, 2017) y síntomas somáticos relacionados con eventos traumáticos (Garnefski, van Rood, de Roos, \& Kraaij, 2017), entre otros.

Un instrumento de amplia difusión para la medición de las ERC es el Cognitive Emotion Regulation Questionnaire (CERQ; Garnefski et al., 2001), el cual cuenta con una versión breve de 18 ítems (CERQ-18) y que ha demostrado adecuadas propiedades psicométricas (Dominguez-Lara \& Merino-Soto, 2015, 2018). Sin embargo, a pesar de la relevancia del constructo para la comprensión de la conducta del adolescente, solo existen los estudios de Garnefski et al. (2001) y de D'Acremont y Van der Linden 
(2007) donde se analizan las propiedades psicométricas del CERQ. Estos estudios presentan limitaciones metodológicas como la utilización del análisis por componentes principales (Dominguez-Lara \& Merino-Soto, 2016), así como derivar puntuaciones generales en base a modelos de segundo orden, en lugar de modelos jerárquicos directos (Canivez, 2016). Otros estudios con muestras latinoamericanas han empleado algunas de las dimensiones originales del CERQ en estudios empíricos con niños o adolescentes (e.g., Andrés, Richaud, Castañeiras, Canet-Juric, \& Rodríguez-Carvajal, 2016), pero no han sido reportadas las bondades psicométricas de esas versiones, por lo que aún puede considerarse un área de vacancia en investigación.

En los estudios instrumentales del CERQ en su versión general llevados a cabo en población adulta, destaca la presencia de elevadas correlaciones interfactoriales. En ese contexto, se ha propuesto un agrupamiento de dimensiones atendiendo a una clasificación a priori de su función en la adaptación de la persona, como la de estrategias apropiadas e inapropiadas (D'Acremont \& Van der Linden, 2007). Sin embargo, existe un planteamiento evolucionista (Medrano et al., 2016) que agrupa las estrategias según su nivel de complejidad. En primer lugar, están las estrategias automáticas (rumiación, catastrofización, autoculpa), que involucran estrategias arcaicas orientadas a la supervivencia del organismo y estructuras subcorticales, por ello resultan de difícil control. Estas estrategias tendrían un nacimiento temprano y común a nivel subcortical, y conjuntamente, aunque de forma más tardía; una contribución de áreas pre-frontales (Hofmann, Ellard, \& Siegle, 2012) que le otorgaría especificidad a cada estrategia automática, por lo cual se presume, desde un punto de vista metodológico, que es mejor representado por un modelo bifactor subyacente, con factores específicos y un factor general (FG) ortogonal.

Por otro lado, están las estrategias elaborativas (aceptación, focalización en los planes, reinterpretación positiva, focalización positiva y poner en perspectiva), cuyo carácter racional exige que la persona despliegue mayores recursos atencionales (Dominguez-Lara \& Medrano, 2016). Cada grupo de estrategias tuvo, y tiene, un rol particular en la evolución humana (Medrano et al., 2016), por lo que denominarlas como inapropiadas o apropiadas sin conocer el contexto en el cual se desarrolla, puede dejar de lado la función que cumple en un momento dado (Dominguez-Lara \& Sánchez-Carreño, 2017). Si bien este modelo fue desarrollado y confirmado en adultos, sería conveniente evaluar si este planteamiento se replica en adolescentes debido a sus características cognitivas (Ahmed, Bittencourt-Hewitt, \& Sebastian, 2015; Rood, Roelofs, Bogels, \& Arntz, 2012), así como la reactividad emocional propia de su condición evolutiva, la cual ejercería una influencia significativa aun cuando son desplegadas las estrategias elaborativas.

Un aspecto clave al momento de obtener evidencias de validez es variar las estrategias empleadas y no restringir los análisis solo a la estructura interna del instrumento (AERA, APA, \& NCME, 2014). En este sentido, se busca relacionar las estrategias medidas con el CERQ18 con otros constructos teóricamente relevantes, y con base en la evidencia previa se considera que un criterio externo de mayor relevancia es el afecto positivo (eutimia) y negativo (distimia) (e.g. Legerstee et al., 2009; Nolen-Hoeksema et al., 2008).

Finalmente, el uso de instrumentos breves en la literatura internacional se ha incrementado debido a sus ventajas comparativas, tanto desde el punto de vista del examinado y del investigador, ya que es posible incluir diferentes instrumentos breves en una sola encuesta así como utilizarlos en estudios nacionales de largo alcance (Rammstead \& Beierlein, 2014). Por ello, sería conveniente analizar la dimensionalidad del CERQ-18 en una población distinta del que fue trabajado inicialmente. En este sentido, en base a la literatura previa, la hipótesis de investigación apunta encontrar una estructura factorial en base a la propuesta de Medrano et al. (2016), que considera el uso de estrategias automáticas y estrategias elaborativas. Además, se espera que estrategias como rumiación o catastrofización tengan una relación directa y significativa con distimia, mientras que estrategias como aceptación o reinterpretación positiva un patrón correlacional similar con eutimia. 


\section{Método}

\section{Diseño}

La presente investigación se rige bajo un diseño instrumental (Ato, López, \& Benavente, 2013), orientado a la exploración de las propiedades psicométricas de un instrumento de medición a través de una evaluación en un momento temporal determinado.

\section{Participantes}

La muestra fue de 255 estudiantes (52\% fueron mujeres) de nivel secundaria de una Institución Educativa de gestión pública del distrito de Ventanilla (Callao, Perú), cuyo nivel socioeconómico es predominantemente mediobajo (incidencia de pobreza 28.5 - 32.9\%; INEI, 2013). El 66.8\% viven con ambos padres y el $26.6 \%$ solo con uno de ellos. El rango de edad estuvo entre $\operatorname{los} 13$ y 19 años $\left(M_{\text {edad }}=15.494\right.$, $\left.D E_{\text {edad }}=1.192\right)$. Respecto al grado de estudio, el $39.9 \%$ pertenecen al tercer año, el $31.4 \%$ al cuarto año y el $28.8 \%$ al quinto año.

El procedimiento de selección de los participantes fue el no probabilístico debido a la accesibilidad y las condiciones para la evaluación como el horario de clase y el tiempo proporcionado para el ingreso a las aulas.

\section{Instrumentos}

Cognitive Emotional Regulation Questionnaire (CERQ-18, Dominguez-Lara \& Merino-Soto, 2015). Este instrumento consta de 18 ítems y cuenta con cinco opciones de respuesta politómica que van desde casi nunca (1) a siempre (5). Los ítems están agrupados en nueve estrategias: Rumiación, Catastrofización, Autoculparse, Culpar a otros, Poner en perspectiva, Aceptación, Focalización positiva, Reinterpretación positiva y Refocalización en los planes; a razón de dos ítems por estrategia.

Inventario de Depresión Estado-Rasgo (IDER; Spielberger, Agudelo, \& Buela-Casal, 2008). En su versión adaptada para adolescentes peruanos (Merino, Pflucker, \& Riaño-Hernández, 2013), está conformado por 20 ítems con cuatro opciones de respuestas que van desde Nada hasta Mucho, agrupados en dos dimensiones: Estado y Rasgo, que a su vez cuentan con dos factores cada una de ellas: eutimia y distimia, que brindan información sobre la experiencia de afecto positivo y negativo, respectivamente. Para el presente estudio solo se utilizó la dimensión Rasgo y sus dos dimensiones (10 ítems) y con los datos obtenidos se realizó previamente un AFC en el que se obtuvo índices de ajuste aceptables (CFI $=.981$, RMSEA $=.056$ [.034-.077], SRMR=.065), una correlación interfactorial teóricamente consistente $(\phi=-.475)$, $\mathrm{y}$ adecuada confiabilidad del constructo $\left(\omega_{\text {eutimia }}=\right.$ $\left..779 ; \omega_{\text {distimia }}=.903\right)$.

\section{Procedimiento}

La presente investigación forma parte de un proyecto de investigación aprobado por el Instituto de Investigación de la Escuela de Psicología la Universidad de San Martín de Porres. Solo fueron incluidos en el estudio a quienes presentaron el consentimiento informado de los padres y/o apoderados, y aceptaban participar a través del llenado del asentimiento informado. De forma complementaria, a quienes mostraban la disponibilidad para el llenado de los cuestionarios.

\section{Análisis de datos}

De forma preliminar fueron evaluadas las características distribucionales de los ítems, concretamente de la asimetría y curtosis, esperando valores entre +/- 1.5 (Pérez \& Medrano, 2010). En cuanto al aspecto estructural, se evaluó cuatro modelos. M1: El modelo original de nueve factores oblicuos (Garnefski et al., 2001). M2: El modelo propuesto por Andrés y colaboradores (Andrés et al., 2016) de cinco factores oblicuos (autoculpa, rumiación, catastrofización, focalización y reinterpretación positiva). M3: un modelo jerárquicos directo (bifactor) de dos factores generales correlacionados (D'Acremont \& Van der Linden, 2007) que agrupan las estrategias apropiadas $\left(\mathrm{FG}_{1} ;\right.$ aceptación, poner en perspectiva, reinterpretación positiva, focalización en los planes y focalización positiva) e inapropiadas $\left(\mathrm{FG}_{2}\right.$; autoculparse, rumiación, catastrofización y culpar a otros). M4: el modelo de corte evolucionista (Medrano et al., 2016) en donde las estrategias automáticas presenta un modelo bifactor en el cual existe un FG y factores específicos ortogonales que involucran a las 
estrategias de autoculparse, rumiación, catastrofización; mientras que las estrategias elaborativas se agrupan sobre la base de un modelo de cuatro factores oblicuos (aceptación, poner en perspectiva, reinterpretación positiva, y focalización en los planes). En cada uno de los modelos se evaluó la normalidad multivariada mediante el coeficiente de Mardia, donde valores superiores a 70 indican un alejamiento de dicha distribución (Rodríguez \& Ruiz, 2008).

El análisis de la estructura interna se realizó mediante un AFC con el programa EQS 6.2. (Bentler \& Wu, 2012). Se utilizó el método de máxima verosimilitud robusto (MVR) y las matrices de correlación policórica debido a la naturaleza ordinal de las opciones de respuesta (Verdam, Oort, \& Sprangers, 2016). El ajuste del modelo fue evaluado según los valores del CFI ( $\geq .95$; Hu \& Bentler, 1999), SRMR ( $\leq .08$; Hu \& Bentler, 1999) y el RMSEA ( $\leq .05$; Steiger \& Lind, 1980).

En el caso de los modelos bifactor, la evaluación de los factores generales $\left(\mathrm{FG}_{1}, \mathrm{FG}_{2}\right)$ se realizó mediante el análisis del ECV (Explained Common Variance) y el ECV-I; donde valores $>.80$ indican influencia significativa del FG sobre el ítem. Del mismo modo, el PUC (Percentage of Uncontaminated Correlations) y el coeficiente omega jerárquico $\left(\omega_{H}\right.$, Zinbarg, Yovel, Revelle, \& McDonald, 2006). Una valoración conjunta indica que valores ECV > .60, PUC >.80 у $\omega_{H}>.70 o$ valores ECV >.70 y PUC >.70 podrían indicar unidimensionalidad estructural (Rodriguez, Reise, \& Haviland, 2016).

Las evidencias de validez interna convergente se obtuvo con la varianza promedio extraída (Average Variance Extracted [AVE]) considerando valores alrededor del .50 como satisfactorios (Fornell \& Larcker, 1981). Las evidencias de validez interna discriminante, se obtuvo comparando el cuadrado de la correlación interfactorial $\left(\varphi^{2} ;\right.$ varianza compartida entre factores) con la AVE implicado en esa asociación, esperando que cada AVE sea mayor que $\varphi^{2}$, lo que permitiría concluir que los dos factores analizados se diferencian satisfactoriamente.

Las evidencias de validez por su relación con otras variables fueron realizadas entre el modelo del CERQ que presente evidencias más adecuadas, y las dimensiones eutimia y distimia

del IDER-rasgo. Las correlaciones >.20 fueron consideradas como significativas (Ferguson, 2009).

Por otro lado, la confiabilidad del constructo fue calculadas a través del coeficiente omega $(\omega$, McDonald, 1999) debido a que es el más recomendado por su precisión (Zinbarg, Revelle, Yovel, \& Li, 2005). La confiabilidad de las puntuaciones fue evaluada por medio del coeficiente $\alpha$ ( $>2$ ítems) y coeficiente AngoffFeldt (Feldt \& Brennan, 1989) para medidas de dos ítems (Dominguez-Lara, Merino-Soto, \& Navarro-Loli, 2016). En cuanto a la interpretación, magnitudes $>.70$ son aceptables (Hunsle \& Marsh, 2008).

\section{Resultados}

\section{Análisis descriptivo}

En la Tabla 1, los resultados indican que los valores de asimetría y curtosis para todos los ítems del CERQ-18 se encuentran entre +/- 1.5, considerado aceptable porque indica una variación leve en la distribución de los datos (Pérez, \& Medrano, 2010) permitiendo la realización del análisis factorial (Ferrando \& Anguiano-Carrasco, 2010).

Tabla 1. Estadísticos descriptivos

\begin{tabular}{|c|c|c|c|c|}
\hline & M & $\mathrm{DE}$ & g1 & g2 \\
\hline$\overline{\text { CERQ1 }}$ & 3.661 & 1.090 & -.382 & -.570 \\
\hline CERQ2 & 2.325 & 1.172 & .580 & -.408 \\
\hline CERQ3 & 2.900 & 1.259 & .212 & -.875 \\
\hline CERQ4 & 3.661 & 1.162 & -.408 & -.762 \\
\hline CERQ5 & 3.727 & 992 & -.278 & -.681 \\
\hline CERQ6 & 3.469 & 1.166 & -.417 & -.535 \\
\hline CERQ7 & 3.266 & 1.120 & -.173 & -.595 \\
\hline CERQ8 & 3.528 & 1.108 & -.399 & -.393 \\
\hline CERQ9 & 3.150 & 1.200 & -.061 & -.757 \\
\hline CERQ10 & 2.959 & 1.082 & .010 & -.448 \\
\hline CERQ11 & 3.066 & 1.193 & -.129 & -.738 \\
\hline CERQ12 & 3.225 & 1.150 & -.067 & -.649 \\
\hline CERQ13 & 2.985 & 1.161 & .029 & -.674 \\
\hline CERQ14 & 2.454 & 1.240 & .541 & -.656 \\
\hline CERQ15 & 4.011 & 1.052 & -.791 & -.224 \\
\hline CERQ16 & 3.435 & 1.177 & -.406 & -.509 \\
\hline CERQ17 & 3.055 & 1.109 & -.077 & -.519 \\
\hline CERQ18 & 3.148 & 1.214 & .052 & -.837 \\
\hline
\end{tabular}


Tabla 2. Análisis factorial confirmatorio, confiabilidad del constructo y validez convergente de M1 y M2

\begin{tabular}{|c|c|c|c|c|c|c|c|c|c|c|c|c|c|c|}
\hline & \multicolumn{9}{|c|}{ M1 } & \multicolumn{5}{|c|}{ M2 } \\
\hline & E1 & E2 & E3 & E4 & E5 & E6 & E7 & E8 & E9 & E1 & E2 & E3 & E7 & E9 \\
\hline CERQ9 & .588 & & & & & & & & & .554 & & & & \\
\hline CERQ17 & .686 & & & & & & & & & .729 & & & & \\
\hline CERQ7 & & .641 & & & & & & & & & .642 & & & \\
\hline CERQ13 & & .708 & & & & & & & & & .706 & & & \\
\hline CERQ3 & & & .741 & & & & & & & & & .721 & & \\
\hline CERQ18 & & & .853 & & & & & & & & & .876 & & \\
\hline CERQ2 & & & & .718 & & & & & & & & & & \\
\hline CERQ14 & & & & .825 & & & & & & & & & & \\
\hline CERQ1 & & & & & .556 & & & & & & & & & \\
\hline CERQ8 & & & & & .942 & & & & & & & & & \\
\hline CERQ4 & & & & & & .457 & & & & & & & & \\
\hline CERQ10 & & & & & & .555 & & & & & & & & \\
\hline CERQ11 & & & & & & & .792 & & & & & & .817 & \\
\hline CERQ16 & & & & & & & .756 & & & & & & .733 & \\
\hline CERQ5 & & & & & & & & .759 & & & & & & \\
\hline CERQ15 & & & & & & & & .676 & & & & & & \\
\hline CERQ6 & & & & & & & & & .625 & & & & & .558 \\
\hline CERQ12 & & & & & & & & & .618 & & & & & .692 \\
\hline$\omega$ & .578 & .625 & .778 & .747 & .736 & .408 & .749 & .68 & .557 & .586 & .625 & .781 & .751 & .63 \\
\hline AVE & .408 & .456 & .638 & .598 & .598 & .258 & .599 & .516 & .386 & .419 & .455 & .643 & .602 & .395 \\
\hline E1 & 1 & .625 & .692 & .094 & .164 & .300 & .040 & .004 & .019 & 1 & .619 & .697 & .029 & .018 \\
\hline E2 & .791 & 1 & .705 & .188 & .000 & .023 & .011 & .000 & .016 & .787 & 1 & 690 & .011 & .012 \\
\hline E3 & .832 & .84 & 1 & .378 & .001 & .027 & .148 & .043 & .110 & .835 & .831 & 1 & .138 & .103 \\
\hline E4 & .307 & .434 & .615 & 1 & .018 & .033 & .203 & .078 & .061 & - & - & - & - & - \\
\hline E5 & .405 & -.006 & .040 & -.135 & 1 & .356 & .151 & .295 & .188 & - & - & - & - & - \\
\hline E6 & .548 & .152 & .165 & -.183 & .597 & 1 & .687 & .532 & .429 & .173 & -.108 & -.372 & 1 & .577 \\
\hline E7 & .2 & -.108 & -.386 & -.451 & .389 & .829 & 1 & .508 & .588 & - & - & - & .76 & - \\
\hline E8 & .064 & -.007 & -.209 & -.281 & .544 & .730 & .713 & 1 & .672 & -.136 & .112 & -.322 & .543 & 1 \\
\hline E9 & -.137 & .127 & -.332 & -.247 & .434 & .655 & .767 & .82 & 1 & .419 & .455 & .643 & .602 & .395 \\
\hline
\end{tabular}

Nota. AVE: Average Variance Extracted; $\omega=$ coeficiente omega; E1=Autoculparse, E2=Rumiación; E3=Catastrofización; E4=Culpar a otros; E5=Aceptación; E6=Poner en perspectiva; E7=Reinterpretación Positiva; E8=Focalización en los planes; E9=Focalización positiva; Bajo la diagonal: correlaciones interfactoriales; Sobre la diagonal: varianza compartida entre factores.

\section{Evidencias de validez con relación a la estructura interna}

En la Tabla 2, se observa que, en cuanto a las evidencias de validez de la estructura interna de M1, los índices de ajuste obtenidos fueron aceptables (CFI=.960, RMSEA $=.054$ [IC90\% $.041-.067]$, SRMR=.060), y todas las cargas factoriales fueron estadísticamente significativas y de magnitud moderada $\left(\lambda_{\text {promedio }}=.694\right)$, con un rango de $\lambda_{\text {mín }}=.457$ (poner en perspectiva) a $\lambda_{\text {máx }}=.942$ (aceptación). Por otro lado, para M2 se obtuvo CFI=.976, RMSEA=.054 (.026-.079), $\mathrm{SRMR}=.051$, con cargas factoriales significativas moderadas $\left(\lambda_{\text {promedio }}=.702\right)$, con un rango de $\lambda_{\operatorname{mín}}=.554 \quad$ (autoculparse) a $\lambda_{\operatorname{máx}}=.876$ (catastrofización). Cabe resaltar que los coeficientes de Mardia fueron de .89 .959 para M1 y de 75.856 para M2, lo que implica un alejamiento leve de la normalidad multivariada.

Respecto a las evidencias de validez interna convergente, en M1 la AVE de autoculparse, rumiación, poner en perspectiva, y focalización positiva, y de $\mathrm{M} 2$, autoculparse, rumiación y focalización positiva, son menores que .50 .
Referente a la validez interna discriminante, en M1, todas las estrategias, excepto culpar a otros y aceptación, presentan valores de la AVE menores al cuadrado de las correlaciones interfactoriales $\left(\varphi^{2}\right)$; y en M2, todas excepto catastrofización.

En el modelo bifactor (M3), este obtuvo índices de ajuste adecuados (CFI=.964, RMSEA $=.044$ [IC90\% .036-.051], SRMR=.085), con un $\lambda_{\text {promedio }}$ de .401 para los factores específicos, y de .575 para $\mathrm{FG}_{1}$ (estrategias inapropiadas) y de .543 para $\mathrm{FG}_{2}$ (estrategias apropiadas) (Tabla 3). Del mismo modo, el coeficiente de Mardia fue de 72.344.

Los índices estadísticos del modelo bifactor indican que los FG explican en mayor proporción la varianza de los ítems. Del mismo modo, las magnitudes de los coeficientes $\omega_{H}$ de los factores específicos son menores en comparación a los del FG. El 50\% de ítems posee índices ECV-I con valores alrededor del .80 , pero la magnitud de la AVE y ECV de los FGs no son lo suficientemente elevados para plantear una interpretación parsimoniosa de dos factores. Por ejemplo, culpar 
Tabla 3. Análisis factorial confirmatorio y validez convergente del modelo bifactor (M3)

\begin{tabular}{|c|c|c|c|c|c|c|c|c|c|c|c|c|}
\hline & E1 & E2 & E3 & E4 & E5 & E6 & E7 & E8 & E9 & $\mathrm{FG}_{1}$ & $\mathrm{FG}_{2}$ & ECV-I \\
\hline CERQ9 & .472 & & & & & & & & & .375 & & .386 \\
\hline CERQ17 & .631 & & & & & & & & & .646 & & .782 \\
\hline CERQ7 & & .626 & & & & & & & & .533 & & .420 \\
\hline CERQ13 & & .232 & & & & & & & & .578 & & .861 \\
\hline CERQ3 & & & -.072 & & & & & & & .710 & & .989 \\
\hline CERQ18 & & & .120 & & & & & & & .902 & & .982 \\
\hline CERQ2 & & & & .870 & & & & & & .366 & & .071 \\
\hline CERQ14 & & & & .476 & & & & & & .487 & & .511 \\
\hline CERQ1 & & & & & .553 & & & & & & .365 & .303 \\
\hline CERQ8 & & & & & .607 & & & & & & .514 & .417 \\
\hline CERQ4 & & & & & & .590 & & & & & .488 & .406 \\
\hline CERQ10 & & & & & & .116 & & & & & .379 & .914 \\
\hline CERQ11 & & & & & & & .608 & & & & .559 & .458 \\
\hline CERQ16 & & & & & & & .359 & & & & .681 & .782 \\
\hline CERQ5 & & & & & & & & .492 & & & .715 & .678 \\
\hline CERQ15 & & & & & & & & .085 & & & .659 & .983 \\
\hline CERQ6 & & & & & & & & & .580 & & .570 & .491 \\
\hline CERQ12 & & & & & & & & & .174 & & .501 & .892 \\
\hline AVE & .169 & .222 & .009 & .491 & .337 & .18 & 249 & .124 & .183 & .358 & .307 & \\
\hline ECV & & & & & & & & & & .615 & .588 & \\
\hline PUC & & & & & & & & & & .857 & .888 & \\
\hline$\omega_{H}$ & .196 & .208 & .000 & .509 & .390 & .172 & .235 & .083 & .169 & .716 & .735 & \\
\hline
\end{tabular}

Nota. AVE=Average Variance Extracted; ECV=Explained Common Variance; PUC=Percentage of Uncontaminated Correlations; $\omega H=$ coeficiente omega jerárquico; FG1 = estrategias inapropiadas; FG2 = estrategias apropiadas; E1= Autoculparse, E2=Rumiación; E3=Catastrofización; E4=Culpar a otros; E5=Aceptación; E6=Poner en perspectiva; E=Reinterpretación Positiva; E8=Focalización en los planes; E9=Focalización positiva.

$a$ otros presenta un $\omega_{\mathrm{h}}$ de una magnitud no despreciable (>.30) y un AVE >.50 que lo diferencia sustantivamente de su FG.

Por último, con respecto al modelo evolucionista, el ajuste para el modelo jerárquico bifactor (estrategias automáticas) fue bueno: CFI=.999; RMSEA=.021 (IC90\% .000-.107), $\mathrm{SRMR}=.035$; teniendo una $\lambda_{\text {promedio }}$ de .415 para los factores específicos y de .626 para el FG. Asimismo, el FG explica una mayor proporción de la varianza lo que se evidencia en que el $70 \%$ de ítems posea valores ECV-I mayores al .80. Además, los valores de la AVE para cada factor específico son menores que 20 y para el FG es de .408 (Tabla 4), lo que resulta en la viabilidad de la interpretación de una sola puntuación. En cuanto al ajuste del modelo de factores oblicuos correspondiente a las estrategias elaborativas, este es aceptable: $\mathrm{CFI}=.966 ; \quad \mathrm{RMSEA}=.080$ (IC90\% .05-.111), SRMR=.056; con cargas factoriales estadísticamente significativas $\left(\lambda_{\text {promedio }}\right.$ $=.684$ ), en el rango de $\lambda_{\text {mín }}=.542$ (poner en perspectiva) a $\lambda_{\text {máx }}=.892$ (reinterpretación positiva) (Tabla 5). Finalmente, el valor de la AVE de poner en perspectiva fue el único menor a .50, y las evidencias de validez discriminante indican que las estrategias de poner en perspectiva y focalización en los planes tienen valores de la AVE menores al cuadrado de las correlaciones interfactoriales $\left(\varphi^{2}\right)$, lo que no establece la diferenciación empírica entre dimensiones y, en consecuencia, la posibilidad de interpretar las dimensiones por separado no es posible, al menos con esas dos dimensiones. De forma similar a los modelos preliminares, el coeficiente de Mardia fue de 77.410.

\section{Evidencias de validez con relación a otras variables}

Dadas las evidencias preliminares, M4 se erige como el modelo con evidencias más robustas. En ese sentido, las asociaciones entre el FG (estrategias automáticas) es directa y significativa con distimia, y observándose correlaciones en dirección opuesta con eutimia (Tabla 4). Del mismo modo, las estrategias elaborativas se asocian de consistentemente y de forma positiva y negativa con eutimia y distimia, respectivamente. (Tabla 5).

Incluye valores cuando des resultados significativos al menos Rpta: no pueden incluirse valores ya que estos figuran en la Tabla. De hacerlo, se redunda en información.

\section{Confiabilidad}

En general, los valores de confiabilidad de constructo se encuentran en el rango de lo aceptable, excepto para la estrategia de poner en 
Tabla 4. Análisis bifactor de M4 (estrategias

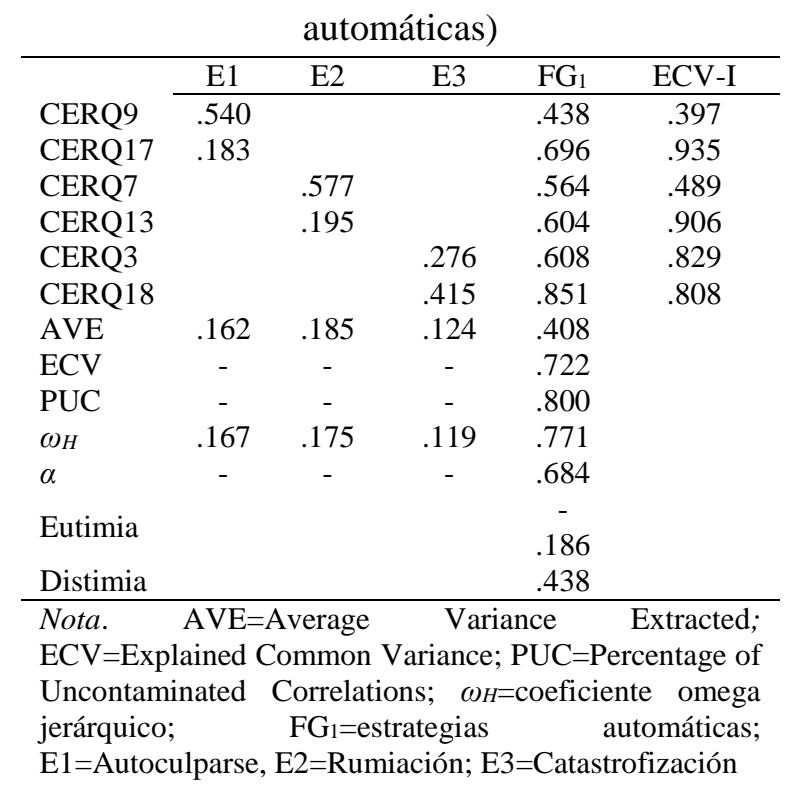

Tabla 5. Análisis factorial confirmatorio de M4 (estrategias elaborativas)

\begin{tabular}{|c|c|c|c|c|}
\hline & E5 & E6 & E7 & E8 \\
\hline CERQ1 & .598 & & & \\
\hline CERQ8 & .876 & & & \\
\hline CERQ4 & & .542 & & \\
\hline CERQ10 & & . 467 & & \\
\hline CERQ11 & & & .671 & \\
\hline CERQ16 & & & .892 & \\
\hline CERQ5 & & & & .759 \\
\hline CERQ15 & & & & .676 \\
\hline$\omega$ & .712 & .406 & .764 & .680 \\
\hline AVE & .562 & .255 & .622 & .512 \\
\hline E5 & 1 & .432 & .181 & .351 \\
\hline E6 & .658 & 1 & .481 & .628 \\
\hline E7 & .426 & .694 & 1 & .558 \\
\hline E8 & .593 & .793 & .747 & 1 \\
\hline$r_{A F}$ & .629 & .370 & .708 & .640 \\
\hline Eutimia & .291 & .351 & .535 & .545 \\
\hline Distimia & -.109 & -.224 & -.280 & -.507 \\
\hline \multicolumn{5}{|c|}{$\begin{array}{l}\text { Nota. AVE=Average Variance Extracted; } \omega \\
=\text { coeficiente omega; } r_{A F}=\text { Coeficiente Angoff-Feldt; } \\
\text { AVE: Average Variance Extracted; E5=Aceptación; } \\
\text { E6=Poner en perspectiva; E7=Reinterpretación } \\
\text { Positiva; E8=Focalización en los planes; Debajo de la } \\
\text { diagonal: correlaciones interfactoriales; Encima de la } \\
\text { diagonal: varianza compartida entre factores. }\end{array}$} \\
\hline
\end{tabular}

perspectiva $(\omega<.70)$ (Tablas 4 y 5$)$. En cuanto a la confiabilidad de las puntuaciones, en general, los valores son cercanos al mínimo aceptable (.70), excepto poner en perspectiva que obtuvo una magnitud bastante baja $(<.40)$.

\section{Discusión}

El presente estudio tuvo como objetivo brindar evidencias de validez relacionadas con la estructura interna y la relación con otras variables de la versión peruana del CERQ-18 (Dominguez-Lara \& Merino-Soto, 2018) en una muestra de adolescentes peruanos en el marco de los planteamientos actuales de validez (AERA, APA, \& NCME, 2014). Cabe resaltar que este es el primer estudio instrumental con muestra latinoamericana en dicha población y donde se evalúan, además del modelo oblicuo, diversos modelos de medición asociados a avances teóricos respecto a las ERC, lo que incluye modelos jerárquicos vinculados a la estructura interna del CERQ mediante metodología recomendada, como son los modelos bifactor que permiten una adecuada separación entre el FG y los específicos para identificar de manera precisa la influencia del FG sobre los ítems (Canivez, 2016; Chen, West, \& Sousa, 2006; Dominguez-Lara \& Rodríguez, 2017; Gignac, 2008).

Se planteó como hipótesis, la replicación de la estructura propuesta por Medrano et al. (2016), y en base a los resultados, se puede inferir que es la más parsimoniosa y verosímil. Esto, porque al analizar M1 y M2, se observan correlaciones interfactoriales que pueden indicar multicolinealidad, además porque los FGs planteados en base a D'Acremont, y Van der Linden (2007) y Garnefski et al. (2001) presentan indicadores que no garantizaron su interpretabilidad de forma similar a los hallazgos en adultos (Dominguez-Lara \& Medrano, 2016, Domínguez-Sánchez, Lasa-Aristu, Amor, \& Holgado-Tello, 2013, Perte \& Miclea, 2011), derivando en un modelo más parsimonioso para viabilizar diversos análisis (Ziegler et al., 2014). Esto tuvo lugar debido a que la estrategia de culpar a otros posee el mayor valor de varianza explicada por el propio factor en comparación a las otras estrategias, y lo diferencia sustantivamente del FG (estrategias inapropiadas).

Estos datos podrían indicar que culpar a otros no tendría la misma connotación desadaptativa en adolescentes, lo que se sustenta además por lo reportado en niños (Orgilés, Morales, FernándezMartínez, Melero, \& Espada, 2018), pese a estar asociada a la vivencia de emociones negativas como la ira (Bennett, Lowe, \& Honey, 2003; Hazebroek, Howells, \& Day, 2001; Kuppens \& Van Mechelen, 2007; Kuppens, Van Mechelen, \& Rijmen, 2008), no parece asociarse significativamente a procesos automáticos porque 
diversos estudios indican que culpar a otros necesita de procesos adicionales como la desconfianza interpersonal y la percepción de una baja valoración personal por parte de los pares (Murray, Griffin, Rose, \& Bellavia, 2003; Orobio de Castro, Veerman, Koops, Bosch, \& Monshouwer, 2002), lo que implica un procesamiento cognitivo para ejecutarse. Estas relaciones pueden ser explicadas porque durante el desarrollo de la adolescencia las estructuras corticales encargadas de funciones ejecutivas continúan en desarrollo (Giedd, 2008; Kuhn, 2006). Todo ello predispone a reacciones emocionales e impulsivas a los adolescentes (Eshel, Nelson, Blair, Pine, \& Ernst, 2007; Maciejewski, van Lier, Branje, Meeus, \& Koot, 2015), en vista que en el proceso de regulación emocional las estructuras corticales prefrontales tiene un rol regulador sobre las estructuras subcorticales (Carver, Johnson, \& Joormann, 2008; Guyer, Silk, \& Nelson, 2016).

Entonces, se puede inferir que las ECRE no pueden ser consideradas arbitrariamente bajo la clasificación de funcional/disfuncional o adaptativa/desadaptativa, ya que pueden tener un rol que esté en función del contexto al que se enfrenta la persona (Aldao, 2013; Gross, 2015; Brockman, Ciarrochi, Parker, \& Kasdan, 2016; Kashdan \& Rottenberg, 2010). Por ejemplo, en el contexto académico, asumir la responsabilidad luego de obtener una mala calificación podría resultar útil para el estudiante a fin de que pueda evaluar su proceder en próximas evaluaciones (Dominguez-Lara \& Sánchez-Carreño, 2017).

Para las evidencias de validez en relación a otras variables, se hipotetizó que estrategias automáticas tengan una relación directa $\mathrm{y}$ significativa con distimia, mientras que estrategias elaborativas un patrón correlacional similar con eutimia. Los resultados obtenidos corroboran dicho patrón de asociación y respaldan los planteamientos teóricos de Medrano et al. (2016) y lo reportado en investigaciones previas (Abela \& Hankin, 2011; D'Acremont \& Van der Linden, 2007; Garnefski et al., 2005; Garnefski \& Kraaij, 2006, 2016; Nolen-Hoeksema et al., 2008).

La relevancia de dicha clasificación es mayor si se toma en consideración que permite una mejor articulación con evidencias provenientes de la neurociencia, la psicología evolucionista y el modelo de procesamiento dual. Tal como proponen Clark y Beck (2012) existiría una doble vía para el procesamiento de la información (un procesamiento automático y otro más controlado). El procesamiento automático se podría explicar sobre la base de dos componentes. En primer lugar, un Factor General Automático que hace un reconocimiento rápido y automático del estímulo cuya función es identificar estímulos relevantes para la supervivencia del organismo reorientando los recursos atencionales hacia esa dirección. Este procesamiento es relativamente indiferenciado, rápido, involuntario e inconsciente; lo que permite reconocer fundamentalmente la valencia $\mathrm{y}$ relevancia personal del estímulo. Luego del reconocimiento inicial de un estímulo relevante para la supervivencia ocurre un segundo estadio de procesamiento de la información donde se despliegan cogniciones más complejas que facilitan una preparación del organismo para garantizar el alcance de metas evolutivas básicas, como en la supervivencia (seguridad), la procreación y la sociabilidad. Por ejemplo, pensamientos primarios que se focalizan en el estímulo considerado peligroso, también pueden producir pensamientos automáticos repetitivos (e.g. rumiación) relacionados con el peligro. De este modo, cuando se activan estas cogniciones automáticas se consumen muchos recursos atencionales del sistema de procesamiento de la información, bloqueando de esta forma modos de pensar más constructivos o reflexivos (i.e. procesamiento elaborativo). De esta forma se explicarían las fallas en la regulación cognitiva de las emociones. Finalmente, entre las limitaciones del estudio se encuentra el procedimiento de selección de participantes y que su número no es representativo de la población de adolescentes peruanos. Además, se recomienda que en futuros estudios se evalúe las propiedades métricas del CERQ y su relación con variables en muestras clínicas de adolescentes. En cuanto a la metodología, se sugiere realizar estudios de invarianza de medición debido a que este procedimiento es condicional para la identificación de diferencias individuales interculturales y de género, lo que puede ser importante porque en los resultados encontrados se ha identificado que la estrategia de culpar a otros no es utilizada por los adolescentes peruanos 
lo que contrasta con lo reportado en estudios de otros países.

En conclusión, se puede afirmar que la estructura interna del CERQ-18 respaldada por el modelo teórico de Medrano et al. (2016) es un instrumento con adecuadas propiedades psicométricas que posibilitaría una caracterización más precisa de los grupos evaluados. Del mismo modo, debido a su extensión es posible utilizarlo en estudios más complejos que busquen analizar, desde un marco explicativo, los antecedentes y consecuentes de los dos conjuntos de estrategias analizadas, tanto a nivel personal, familiar, social y académico, a modo de replicación de lo hallado en otros contextos latinoamericanos (Andrés et al., 2016). Con todo, dados los indicadores de confiabilidad, no se recomienda su uso para toma de decisiones a nivel individual.

\section{Referencias}

Abela, J., \& Benjamin, H. (2008). Handbook of Depression in Children and Adolescents. New York: Guilford Press.

Ahmed, S. P., Bittencourt-Hewitt, A., \& Sebastian, C. L. (2015). Neurocognitive bases of emotion regulation development in adolescence. Developmental Cognitive Neuroscience, $\quad 15, \quad 11-25$. doi:10.1016/j.den.2015.07.006.

Aldao, A. (2013). The future of emotion regulation research: Capturing context. Perspectives on Psychological Science, 8, 155-172.

http://dx.doi.org/10.1177/1745691612459518

American Educational Research Association, American Psychological Association, \& National Council on Measurement in Education (2014). Standards for educational and psychological testing. Washington, DC: American Psychological Association.

Andrés, M. L., Richaud, M .C., Castañeiras, C., Canet-Juric, L., \& Rodríguez-Carvajal, R. (2016). Neuroticism and depression in children: the role of cognitive emotion regulation strategies. The Journal of Genetic Psychology, 177(2), 55-71.

doi:10.1080/00221325.2016.1148659
Ato, M., López, J., \& Benavente, A. (2013). Un sistema de clasificación de los diseños de investigación en psicología. Anales de Psicología, 29(3), 1038-1059. doi: http://dx.doi.org/10.6018/analesps.29.3.17851 1

Bennett, P., Lowe, R., \& Honey, K. L. (2003). Appraisals, core relational themes, and emotions: A test of the consistency of reporting and their associations. Cognition and Emotion, 17, 511-520.

Bentler, P. M., \& Wu, E. J. C. (2012). EQS 6.2 for windows [Statistical Program]. Encino, CA: Multivariate Software, Inc.

Brockman, R., Ciarrochi, J., Parker, P., \& Kashdan, T. B. (2016). Emotion regulation strategies in daily life: Mindfulness, cognitive reappraisal and emotion suppression. Cognitive Behaviour Therapy, 46(2). doi:10.1080/16506073.2016.1218926

Burnett, S., \& Blakemore, S. J. (2009). The development of adolescent social cognition. Annals of the New York Academy of Sciences, 1167(1), 51-56. doi:10.1111/j.1749-6632.2009.04509.x

Canivez, G. L. (2016). Bifactor modeling in construct validation of multifactored tests: Implications for multidimensionality and test interpretation. In K. Schweizer \& C. DiStefano (Eds.), Principles and methods of test construction: Standards and recent advancements (pp. 247-271). Gottingen, Germany: Hogrefe.

Carver, C. S., Johnson, S. L., \& Joormann, J. (2008). Serotonergic function, two-mode models of self-regulation, and vulnerability to depression: What depression has in common with impulsive aggression? Psychological Bulletin, 134, 912-943. doi:https://doi.org/10.1037/a0013740

Chen, F. F., West, S. G., \& Sousa, K. H. (2006). A comparison of bifactor and The anxiety and worry workbook: The cognitive behavioral solution second-order models of quality of life. Multivariate Behavioral Research, 41(2), 189-225. doi:10.1207/s15327906mbr4102_5

Clark, D. A., \& Beck A. T. (2012). The anxiety and worry workbook: The cognitive behavioral solution. New York: The Guilford Press. 
D'Acremont, M., \& Van der Linden, M. (2007). How is impulsivity related to depression in adolescence? Evidence from a French validation of the cognitive emotion regulation questionnaire. Journal of Adolescence, 30, 271282. doi:10.1016/j.adolescence.2006.02.007

Denny, B. T., Silvers, J. A., \& Ochsner, K. N. (2009). How we heal what we don't want to feel: neural architecture of emotion regulation. In A. M. Kring \& D. M. Sloan (Eds.), Emotion regulation and psychopathology: A transdiagnostic approach to etiology and treatment (pp. 59-78). New York: Guilford Press.

Dominguez-Lara, S., \& Rodriguez, A. (2017). Índices estadísticos de modelos bifactor. Interacciones, $\quad 3(2), \quad 59-65$. doi:10.24016/2017.v3n2.51

Dominguez-Lara, S., \& Medrano, L. (2016). Propiedades psicométricas del Cognitive Emotional Regulation Questionnaire (CERQ) en estudiantes universitarios de Lima. Psychologia. Avances en la disciplina, 10(1), 53-67.

Dominguez-Lara, S., \& Merino-Soto, C. (2016). Sobre o uso do Little Jiffy na validação dos testes: Comentários a Ávila e colaboradores. Jornal Brasileiro de Psiquiatria, 65(2), 196197.

Dominguez-Lara, S., \& Merino-Soto, C. (2015). Una versión breve del Congnitive Emotional Regulation Questionaire: Análisis estructural del CERQ-18 en estudiantes universitarios limeños. Revista Peruana de Psicología y Trabajo Social, 4(1), 25-36.

Dominguez-Lara, S., \& Merino-Soto, C. (2018). Cognitive Emotional Regulation Questionnaire-18 en universitarios: Evidencias de validez convergente y discriminante. Revista Iberoamericana de Diagnóstico y Evaluación - e Avaliação Psicológica, 47(2), 171-184. doi:10.21865/RIDEP47.2.12.

Dominguez-Lara, S., \& Sánchez-Carreño, K. (2017). Uso de estrategias cognitivas de regulación emocional ante la desaprobación de un examen: El rol de la autoeficacia académica en estudiantes universitarios. Psychologia, 11(2), 99-112. doi:10.21500/19002386.2716
Dominguez-Lara, S., Merino-Soto, C., \& Navarro-Loli, J. (2016). Estimación de la Confiabilidad en Mediciones de dos ítems: El Coeficiente Angoff-Feldt. Revista Digital de Investigación en Docencia Universitaria, 10(1), 34-40. doi:http://dx.doi.org/10.19083/ridu.10.46

Domínguez-Sánchez, F., Lasa-Aristu, A., Amor, P., \& Holgado-Tello, F. (2013). Psychometric properties of the Spanish version of the Cognitive Emotion Regulation Questionnaire. Assessment, 20(2), 253-261. doi:10.1177/1073191110397274.

Erskine, H .E., Baxter, A. J., Patton, G., Moffitt, T. E., Patel, V., Whiteford, H. A., \& Scott, J. G. (2016). The global coverage of prevalence data for mental disorders in children and adolescents. Epidemiology and Psychiatric Sciences, 26(4), 395-402.

doi:http://dx.doi.org/10.1017/S204579601500 1158

Eshel, N., Nelson E. E., Blair, R. J., Pine, D. S., \& Ernst, M. (2007). Neural substrates of choice selection in adults and adolescents: Development of the ventrolateral prefrontal and anterior cingulated cortices. Neuropsychologia, 45, 1270-1279. doi:10.1016/j.neuropsychologia.2006.10.004

Feldt, L. S., \& Brennan, R. L. (1989). Reliability. En Linn, R. L. (Ed). En Educational Measurement (pp. 105-146). New York: Macmillan.

Ferguson, C. J. (2009). An effect size primer: a guide for clinicians and researchers. Professional Psychology: Research and Practice, $\quad 40(5), \quad 532-538$. doi:10.1037/a0015808.

Ferrnando, P. J., \& Anguiano-Carrasco, C. (2010). El análisis factorial como técnica de investigación en psicología. Papeles del Psicólogo, 31(1), 18-33.

Fornell, C., \& Lacker, D. (1981). Evaluating structural equation models with unobservable variables and measurement error. Journal of Marketing Research, 18(1), 39-50. doi:http://dx.doi.org/10.2307/3151312

Garnefski, N., \& Kraaij, V. (2006). Relationship between cognitive emotion regulation strategies and depressive symptoms: A comparative study of five specific samples. Personality and Individual Differences, 40, 
1659-1669.

doi:https://doi.org/10.1016/j.paid.2005.12.009

Garnefski, N., \& Kraaij, V. (2007). The cognitive emotion regulation questionnaire. European Journal of Psychology Assessment, 23, 141149. doi:10.1027/1015-5759.23.3.141

Garnefski, N., \& Kraaij, V. (2016). Specifity of relations between adolescent's cognitive emotion regulation strategies and symptoms of depression and anxiety. Cognition and Emotion. doi:10.1080/02699931.2016.1232698

Garnefski, N., Kraaij, V., \& Spinhoven, P. (2001). Negative life events, cognitive emotion regulation and emotional problems. Personality and Individual Differences, 30, 1311-1327.

doi:https://doi.org/10.1016/S01918869(00)00113-6

Garnerfski, N., van Rood, Y., de Roos, C., \& Kraaij, V. (2017). Relationships between traumatic life events, cognitive emotion regulation strategies, and somatic complaints. Journal of Clinical Psychology in Medical Settings, 24(2), 122-151. doi:10.1007/s10880-017-9494-y.

Garneski, N., Kraaij, V., \& van Etten, M. (2005). Specificity of relations between adolescent's cognitive emotion regulation strategies and Internalizing and Externalizing psychopathology. Journal of Adolescence, 28, 619-631.doi:10.1016/j.adolescence.2004.12.009

Giedd, J. N. (2008). The teen brain: Insights from neuroimaging. Journal of Adolescent Health, 42, 335-343.

Gignac, E. G. (2008). Higher - order models versus direct hierarchical models: $g$ as superordinate or breadth factor? Psychology Science Quarterly, 50(1), 21-43.

Gross, J. J. (2015). Emotion regulation: Current status and future prospects. Psychological Inquiry, $\quad 26(1), \quad 1-26$. doi:http://dx.doi.org/10.1080/1047840X.2014. 940781

Guyer, J., Silk, E. E., \& Nelson, A. E. (2016). The neurobiology of the emotional adolescent: From the inside out. Neuroscience and Biobehavioral Reviews, 70, 74-85.

doi:https://doi.org/10.1016/j.neubiorev.2016.0 6.043
Hazebroek, J. F., Howells, K., \& Day, A. (2001). Cognitive appraisals associated with high trait anger. Personality and Individual Differences, 30(1), 31-45. doi:10.1016/S0191-8869(00)00007-6

Hofmann, S. G., Ellard K. K., \& Siegle G. J. (2012). Neurobiological correlates of cognitions in fear and anxiety: A CognitiveNeurobiological Information Processing Model. Cognition \& Emotion, 26(2), 282-299. doi:http://dx.doi.org/10.1080/02699931.2011. 579414

Hu, L. T., \& Bentler, P. M. (1995). Evaluating model fit. In R. H. Hoyle, Structural equation modeling: Concepts, issues, and applications (pp. 76-99). Thousand Oaks, CA: Sage.

Hunsley, J., \& Marsh, E. J. (2008). Developing criteria for evidence-based assessment: An introduction to assessment that work. In J. Hunsley \& E. J. Marsh (Eds.) A guide to assessments that work (pp. 3-14). Oxford: Oxford University Press.

Instituto Nacional de Estadística e Informática (2013). Mapa de pobreza provincial y distrital. Recuperado desde https://www.inei.gob.pe/media/MenuRecursiv o/publicaciones_digitales/Est/Lib1261/Libro.p df

Kashdan, T. B., \& Rottenberg, J. (2010). Psychological flexibility as a fundamental aspect of health. Clinical Psychology Review, 30, 865-878. doi:10.1016/j.cpr.2010.03.001

Kraaij, V., Garnerfski, N., de Wilde, E. J., Dijkstra, A., Gebhardt, W., Maes, S., \& Ter Doest, L. (2003). Negative life events and depressive symptoms in late adolescence: Bonding and cognitive coping as vulnerability factors? Journal of Youth and Adolescence, 32(3), 185-193.

Kuhn, D. (2006). Do cognitive changes accompany developments in the adolescent brain? Perspectives on Psychological Science, 1, 59-67.

Kuppens, P., \& Van Mechelen, I. (2007). Interactional appraisal models for the anger appraisals of threatened serl-esteem, otherblame, and frustration. Cognition and Emotion, 21(1), 56-77. doi:10.1080/02699930600562193 
Kuppens, P., Van Mechelen, I., \& Rijmen, F. (2008). Toward disentangling sources of individual differences in appraisal and anger. Journal of Personality, 76(4), 969-1000. doi:10.1111/j.1467-6494.2008.00511.x

Legerstee, J. S., Garnefski, N., Jellesma, F. C., Verhulst, F. C., \& Utens, E. M. W. J. (2010). Cognitive coping and childhood anxiety disorders. Euopean Child \& Adolescent Psychiatry, 19, 143-150. doi:10.1007/s00787-009-0051-6

Maciejewski, D. F., van Lier, P. A., Branje, S. J., Meeus, W. H., \& Koot, H. M. (2015). A 5year longitudinal study on mood variability across adolescence using daily diaries. Child Development, $\quad 86, \quad 1908-921$. doi:https://doi.org/10.1111/cdev.12420

McDonald, R. P. (1999). Test theory: A unified treatment. Mahwah: Lawrence Erlbaum Associates, Inc.

Medrano, L. A., Muñoz-Navarro, R., \& CanoVindel, A. (2016). Procesos cognitivos y regulación emocional: Aportes desde una aproximación psicoevolucionista. Ansiedad y Estrés, 22, 47-54. doi:http://dx.doi.org/10.1016/j.anyes.2016.11. 001

Merino, C., Pflucker, D., \& Riaño-Hernández, D. (2012). Análisis factorial exploratorio del Inventario de Depresión Estado-Rasgo (STDEP) en adolescentes. Revista DiversitasPerspectiva en Psicología, 8(2), 319-330.

Mihalca, A. M., \& Tarnavska, Y. (2013). Cognitive emotion regulation strategies and social functioning in adolescents. ProcediaSocial and Behavioral Sciences, 82, 574-579. doi:10.1016/j.sbspro.2013.06.312

Murray, S. L., Griffin, D. W., Rose, P., \& Bellavia, G. M. (2003). Calibrating the sociometer: The relational contingencies of self-esteem. Journal of Personality and Social Psychology, 85, 63-84.

Nolen-Hoeksema, S., Wisco, B. E., \& Lyubomirsky, S. (2008). Rethinking rumination. Perspectives on Psychological Science, 3, 400-424. doi:10.1111/j.1745-6924.2008.00088.x.

Orgilés M., Morales, A., Fernández-Martínez, I., Melero, S., Espada, J. P. (2018). Validation of the version of the Cognitive Emotion
Regulation Questionnaire for Spanish. Journal of Child Health Care. doi:10.1177/1367493518777306

Orobio de Castro, B., Veerman, J. W., Koops, W., Bosch, J. D., \& Monshouwer, H. J. (2002). Hostile attribution of intent and aggressive behavior: A meta-analysis. Child Development, 73, 916-934.

Pen, B., Jin, J., WenHan, Y., Xiu-Hong, L., \& YuSui, C. (2015). Violence-related behaviors among adolescents and its association with cognitive emotion regulation strategies. World Journal of Pediatrics, 12(1), 82-87. doi:10.1007/s12519-015-0014-6

Pérez, E. R., \& Medrano, L. (2010). Análisis factorial exploratorio: Bases conceptuales y metodológicas. Revista Argentina de Ciencias del Comportamiento, 2(1), 58-66.

Raes, F., Griffith, J. W., \& Van der Gucht, K. (2014). School-based prevention and reduction of depression in adolescents: A cluster-randomized controlled trial of a mindfulness group program. Mindfulness, 5(5), 477-486. doi:10.1007/s12671-013-0202-1

Raimundi, M. J., Molina, M. F., HernándezMendo, A., \& Schmidt, V. (2017). Adaptación argentina del Inventario de Fortalezas en adolescentes (VIA-Youth): Propiedades psicométricas y alternativas para su factorización. Revista Iberoamericana de Diagnóstico y Evaluación - e Avaliação Psicológica, 45(3), 159-174. doi:https://doi.org/10.21865/RIDEP45.3.13

Rammstead, B., \& Beierlein, C. (2014). Can't we make it any shorter? The limits of personality assessment and ways to overcome them. Journal of Individual Differences, 35(4), 212220.

doi:https://doi.org/10.1027/16140001/a000141.

Rodriguez, A., Reise, S.P., \& Haviland, M.G. (2016). Evaluating bifactor models: Calculating and interpreting statistical indices. Psychological Methods, 21(2), 137-150. doi:10.1037/met0000045

Rodriguez, M., \& Ruiz, M. (2008). Atenuación de la asimetría y de la curtosis de las puntuaciones observadas mediante transformaciones de variables: Incidencia 
sobre la estructura factorial. Psicológica, 29, 205-227.

Rood, L., Roelofs, J., Bögels, S. M., \& Arntz, A. (2012). The effects of experimentally induced rumination, positive reappraisal, acceptance, and distancing when thinking about a stressful event on affect states in adolescents. Journal of Abnormal Child Psychology, 40(1), 73-84. doi:10.1007/s10802-011-9544-0

Seiffge-Krenke, I. (2000). Causal links between stressful events, coping style, and adolescent symptomatology. Journal of Adolescence, 23(6), 675-691. doi:10.1006/jado.2000.0352

Spielberger, C. D., Agudelo, D., \& Buela-Casal, G. (2008). Inventario de Depresión Estado/Rasgo (IDER). Madrid: TEA Ediciones.

Steiger, J. H., \& Lind, J. C. (1980, May). Statistically based tests for the number of common factors. Paper presented at the annual meeting of the Psychometric Society, Iowa City, IA.

Tan, Y., Xin, X., Wang, X., \& Yao, S. (2017). Cognitive emotion regulation strategies in Chinese adolescents with overweight and obesity. Childhood Obesity. doi:10.1089/chi.2017.0123

Verdam, M. G., Oort, F. J., \& Sprangers, M. A. (2016). Using structural equation modeling to detect response shifts and true change in discrete variables: An application to the items of the SF-36. Quality of Life Research, 25(6), 1361-1383. doi:10.1007/s11136-015-1195-0

Wante, A., Van Beveren, M., Theuwis, L., \& Braet, C. (2017). The effects of emotion regulation strategies on positive and negative affect in early adolescents. Cognition and Emotion. doi:10.1080/02699931.2017.1374242

Yu-Ning, C., Shu-Qiao, Y., \& Xiu-Hong, X. (2017). Cognitive emotion regulation strategies among Chinese adolescents having unprotected sex: A latent profile analysis. Psychology, Health \& Medicine. doi:10.1080/13548506.2017.1325506

Ziegler, M., Kemper, C. J., \& Kruyen, P. (2014). Short Scales - Five Misunderstandings and Ways to Overcome Them. Journal of Individual Differences, 35(4), 185-189. doi:10.1027/1614-0001/a000148
Zinbarg, R. E., Revelle, W., Yovel, I., \& Li, W. (2005). Cronbach's, $\alpha$ Revelle's $\beta$ and McDonald's $\omega$ н: Their relations with each other and two alternative conceptualizations of reliability. Psychometrika, 70(1), 123-133. doi:10.1007/s11336-003-0974-7

Zinbarg, R. E., Yovel, I., Revelle, W., \& McDonald, R. P. (2006). Estimating generalizability to a latent variable common to all of scale's indicators: A comparison of estimators for $\omega \mathrm{h}$. Applied Psychological Measurement, $\quad 30(2), \quad$ 121-144. doi:https://doi.org/10.1177/0146621605278814 